\title{
Helping asthmatic children through bonding therapy
}

\author{
Antonio Madrid* and Nicholas Bennett \\ Redwood Psychology Clinic, Monte Rio, USA
}

\begin{abstract}
Disruptions in maternal-infant bonding are shown to be the mediating variable between maternal distress and the subsequent expression of childhood asthma. When bonding is repaired, it seems that children's asthmatic symptoms diminish or remit. This study evaluated 16 asthmatic children before and after their mothers were treated with bonding therapy. Fourteen improved on 11 measures, including reduction in STEP classification system and medication use. Thirteen children were able to stop all medications. The links between bonding disruptions, stress, and airway inflammation are discussed.
\end{abstract}

\section{Introduction}

Asthma affects more children's lives than any other chronic illness [1]. In 2008, just over 10 million children within the U.S were reported as ever having had a diagnosis of asthma [2]. It is the third-ranking cause of hospital admissions in children under 15 [3]. The financial cost in America in 2007 was $\$ 56$ billion in health costs, lost school and work days, and early deaths [4]. Asthma is responsible for 14 million missed school days annually [5] - more than any other chronic illness.

Since childhood asthma has reached epidemic proportions and has major public health and financial impacts, researchers have been attempting to identify risk factors for the development of this disease. For several years a significant focus of attention has revolved around the connection between maternal distress and the subsequent development of asthma children.

Mrazek, et al. [6], at the National Jewish Center for Immunology and Respiratory Medicine in Denver, studied 150 children whose mothers were asthmatic. They found a link between early problems in coping/parenting and the subsequent expression of asthma. Klinnert, et al. [7] surprised by this finding, noted that this link between parental stress in care-giving and the subsequent development of asthma was the first documented report of such a connection.

Maternal stress is known to be caused by many factors: Cesarean section deliveries, maternal health issues, psychological problems, maternal despondency, to list a few. Cesarean section deliveries and emergency cesarean sections have linked to childhood asthma. A Finnish study of 60,000 births found that mothers who delivered by cesarean sections were $50 \%$ more likely to have a child who later developed asthma [8]. Emergency cesarean section deliveries (even more stressful) predictably raised the asthma rate up to $60 \%$. This finding was replicated by Roduit, et al. [9] who studied 2,917 children.

Annesi-Maesano, et al. [10], using a British cohort of 2583 mothers, investigated whether in utero and perinatal influences contribute to the development and severity of asthma in childhood. Childhood asthma was more frequently reported by mothers when there had been health complications during pregnancy, labor, or delivery, or when the child was ill during the first week of life. She concluded that there is evidence that in utero and perinatal factors may increase the risk of developing asthma. Similarly, a Norwegian study of over 1.5 million mothers and 5,938 asthmatic children found that many types of pregnancy complications represented a risk factor for the development of asthma in the offspring [11].

Kozyrskyj, et al. [12] studied healthcare records of 13,907 children and their mothers from Manitoba databases. Healthcare or prescription medication for depression or anxiety was used to define maternal distress, and asthma status was determined from the children's asthma prescription records. They found that risk for childhood asthma was increased among children who were exposed to continued maternal distress from birth until age 7 years. Similarly, a Puerto Rican study concluded that maternal depressive symptoms were associated with an increased risk of asthma hospitalizations at age 1 year [13].

Mother-child interactions have been shown to predict the development of asthma in the child by school age. Mantymaa, et al. [14] showed that psychological stress is associated with physical illnesses like asthma or infection. As mentioned above, Klinnert, et al. [7] investigated many factors associated with childhood asthma and found that global assessment of parenting problems was a predictor of the development of asthma by age 3. In that same vein, Wright, et al. [15] found that greater levels of caregiver-perceived stress at 2 to 3 months was associated with increased risk of subsequent repeated wheeze among children during the first 14 months of life. Further, they found that prenatal stress was associated with altered innate and adaptive immune responses, concluding that stress-induced perinatal immunomodulation may impact the expression of allergic disease in these children [16].

Cassibba, et al. [17] found that children affected by asthmatic bronchitis were less securely attached than healthy comparisons. These children showed less harmonious and comfort seeking behaviors than healthy children, indicating insecure attachment.

${ }^{*}$ Correspondence to: Antonio Madrid, Redwood Psychology Clinic, Monte Rio, USA, E-mail: madrid@sonic.net

Key words: asthma, bonding therapy, maternal-infant

Received: January 10, 2021; Accepted: January 19, 2021; Published: January 22, 2021 
Three studies investigated the relationship between pediatric asthma and the difficulties that a mother experiences in bonding with her baby. Using the "maternal-infant bonding" paradigm of Klaus and Kennell [18], these studies looked at the incidence of bonding problems within pediatric asthma populations as compared to well-baby groups. Klaus and Kennell had previously demonstrated that a mother's love for her infant will likely be disrupted if the mother and infant were separated at birth or if the mother was experiencing some great stress or trauma in her life during the pregnancy or the perinatal period.

In the first of these studies Feinberg [19] showed that bonding disruptions occurred three times more frequently in mothers of asthmatics than in mothers of well-babies ( $84 \%$ vs $24 \%)$. Schwartz [20] found almost identical numbers: $86 \%$ vs $29 \%$.

Pennington [21] in the third study found that four "non-bonding events" were most predictive of asthma: delay in holding the baby, family death in first year, emotional problems during pregnancy, and maternal emotional problems in the first year. He concluded that bonding disruptions appear to be the mediating variable that links pediatric asthma with the various maternal factors and stressors that have been identified by numerous researchers.

If this is the case-that bonding disruptions are the link between all of these maternal stressors and pediatric asthma-the next question logically surfaces: "What happens if the mother becomes bonded to her asthmatic child?"

From our clinical experience over 15 years, we had observed that a child's asthma seemed to improve once a disrupted bond between a mother and child was repaired. We subjected this observation and hypothesis to two pilot clinical trials. In these studies, each of the mothers was treated with a therapy that focused entirely on processing the traumas surrounding the birth of her child and then creating an imagined idealized birth. We hypothesized that this would repair the disrupted maternal-infant bond and would have an impact on the child's asthma.

The first study involved six mother-child pairs [22]. Five of the six children, including two infants, experienced complete or nearly complete remission from asthma symptoms as measured across 18 variables. In the second study [23], asthma symptoms improved in 12 of 15 children. Eight of the 10 children who were taking medication no longer needed to continue them.

The present study continues the investigation of the hypothesis that children's breathing problems can be improved by working with the mothers to repair the broken maternal-infant bond. This study focuses on the use of measures that are more objective.

\section{Method}

\section{Sample}

Eighteen asthmatic children and their mothers were identified for this study, from responses to newspaper and magazine articles, posted flyers and mailings, and referrals from other therapists. There were three criteria for inclusion in the sample. (1) The child's asthma needed to be diagnosed by a physician. (2) The mother had to be psychologically stable, having a GAF score of 70 or better. GAF (Global Assessment of Functioning) is a scale from the Diagnostic and Statistical Manual, used by mental health workers to assess psychological disorders [24]. (3) The mother needed to have a pre or perinatal history that met the criteria for non-bonding.
The diagnosis of asthma is always problematic because asthma is not a homogeneous heterogeneous condition and is often over-diagnosed [25]. While asthma is a disease consisting of airway constriction and inflammation, it is not unusual for viral wheezing, atopic reactivity, and bronchial inflammation to be diagnosed as asthma [26]. Rather than entering into a debate about the accuracy of the diagnosis of our subjects, we simply decided to use a physician's diagnosis as the criterion for admission into the cohort.

Of the original 18 subjects, one was eliminated because the mother did not meet the GAF standard, and one was eliminated because the therapy deviated from the prescribed standard. All of the mothers in the initial sample had prenatal or perinatal histories consistent with problems in bonding, i.e., there was evidence of either separation at birth or maternal trauma during pregnancy or soon afterwards.

The remaining 16 children ranged in age from 9 months to 19 years. All were under the care of a physician and all were taking medication.

\section{Procedures}

Following the mother's recruitment into the study, an initial interview was conducted either in person or by telephone. The Maternal-Infant Bonding Survey (MIBS) [27] was administered to determine if she met the criterion of a mother with disrupted bonding. The MIBS (see Appendix A), a survey of events which have been linked to bonding disruptions, was developed from the work of Klaus and Kennell [18]. asked:

The mother was then given a questionnaire (see Appendix B) which

- The age of her child when asthma was first diagnosed and the circumstances under which it was diagnosed;

- The current age of the child;

- The way the child's asthma presented itself, including triggers;

- Her assessment of the severity of her child's asthma; and

- Specific measures of her child's condition (described in the Measures section below).

She was then administered the Beck Depression Inventory.

After being given a description of the study, including the treatment proposed, the mothers began treatment, described in the Treatment section below.

\section{Measures}

Eleven variables were used to measure each child's condition before and six months after treatment.

1. Asthma Monitor-a five question instrument which assesses current asthma severity. Each answer was scored from 0 to 4 . The five scores were then added for the child's total score. Higher numbers indicate greater severity. Total scores range from 0 to 20. (See Appendix B.)

2. Step-used by the National Institutes of Health ( U.S. Department of Health and Human Services, 2003) [28] to determine asthma severity and to design treatment protocol (see Appendix B). Two questions are asked about "daytime symptoms" and "night time symptoms," which place the child into one of five Steps or categories:

\section{Steps or Categories}

No Asthma Symptoms (0) 
Mild Intermittent (1)

Day Time Symptoms: equal to or less than 2 days/week

Night Time Symptoms: equal to or less than 2 night/week

Mild Persistent (2)

Day Time symptoms: greater than twice/week but less than once daily

Night time Symptoms: greater than 2 nights/month

Moderate Persistent (3)

Day Time Symptoms: daily

Night Time Symptoms: more than 1 night/week

Severe Persistent (4)

Day Time Symptoms: continual

Night time Symptoms: frequent

3. Doctor Visits - the number of unscheduled visits during the last 6 months.

4. Emergency Room Visits - the number of emergency room visits during the last 6 months.

5. Hospital stays - the number of admissions and the total days during the prior 6 months.

6. Oral steroids - the number of courses and total number of days during the prior 6 months.

7. Days housebound - the number of days that the child was kept from school or stayed in bed or could not go outside and play during the prior 6 months.

8. Asthma symptoms with exercise-whether the child wheezes when, he is active, rated on a four point scale from Never (0) to Always (3).

9. Overall Health-the mother's perception of the child's general health. Scored: 0 to 4 .

10. Energy Level - the mother's perception of the child's vitality. Scored: 0 to 4 .

11. Medications - a list of the medications that the child was currently taking.

All mothers were asked to take the Beck Depression Inventory-II (BDI) immediately before treatment and 6 months following treatment. The BDI is a 21 item, multiple choice, self-report inventory, widely used by mental health professionals to assess the severity of a patient's depression [29]. Each person's total score can range from 0 to 63 , with 0-5 considered a low score, 6-14 a medium score, and 15-and-above a high score.

\section{Treatment}

Each mother was seen in therapy, using a protocol designed to repair the disrupted bond that existed at or near the time of her child's birth. The treatment used a three-phase approach.

1. Phase One: With the mother, identify all Non-Bonding Events (NBE) i.e., the events or situations that interfered with bonding. This was accomplished using the Maternal Infant Bonding Survey and by discussing with the mother her pregnancy and the birth of her child.
2. Phase Two: Afford the mother an opportunity with hypnosis to process each NBE using until the impact of that event was no longer recorded as painful or disturbing. A detailed description of this procedure can be found in American Journal of Clinical Hypnosis [30]. Other interventions besides hypnosis can be used, such as EMDR, art work, Gestalt Therapy, psychodrama [31].

3. Phase Three: Help the mother to imagine a new birth experience, without the interference of the NBE.

Most mothers were able to satisfactorily complete the treatment in three sessions or less.

\section{Results}

Paired-samples t-tests were used to assess improvement in 11 variables after Bonding Therapy. A one-sided $\mathrm{P}$ value $<0.05$ was considered statistically significant. Means are reported \pm 1 SD.

A total of 16 patients were enrolled into the study. Patients ranged in age from 0.75 to 19 years (mean age ranged from $8.0 \pm 4.7$ years). Age of asthma diagnosis ranged from 0.2 to 9 years (mean age ranged from $4.0 \pm 3$ years). Fifty-six percent of the patients were male.

The results of Maternal-Infant Bonding Therapy indicated significant improvement in children's asthma following treatment of the mothers to improve bonding (Table 1).

An analysis of each of the variables used to measure the effects of treatment herein follows.

\section{Measures}

Asthma Monitor, a measure for assessing asthma severity, was collected for 15 patients. There was significant improvement in the pre-treatment $6.7 \pm 4.4$ versus post-treatment $1.8 \pm 2.5$ average scores $(\mathrm{P}<0.001)$. Every child except one of the two oldest children improved in his Asthma Monitor score.

STEP, a national standard for assessing asthma severity, registered significant improvement in the 15 patients measured, with average pretreatment scores of $3.4 \pm 1.4$ compared with the post-treatment average $0.7 \pm 1.1(\mathrm{P}<0.001)$. The average score before treatment was between Moderate Persistent to Severe Persistent; after treatment the average score was between No Asthma to Mild Intermittent. Before treatment, there were no children in the No Asthma class: post treatment there were 9 children in this class. Before treatment there were 8 children with Severe Persistent asthma, and by the end of treatment, there were none in this category. Except for the two oldest children, every child's STEP classification improved after treatment.

Table 1. Maternal-infant bonding results

\begin{tabular}{|l|c|c|c|}
\hline \multicolumn{1}{|c|}{ Variable } & Pre-treatment & Post-treatment & p-value \\
\hline Asthma monitor & $6.7 \pm 4.4$ & $1.8 \pm 2.5$ & $<0.001$ \\
\hline STEP & $3.4 \pm 1.4$ & $0.7 \pm 1.1$ & $<0.001$ \\
\hline Doctor visits & $1.8 \pm 1.8$ & $0.2 \pm 0.5$ & $<0.001$ \\
\hline ER visits & $0.4 \pm 1.0$ & $0.0 \pm 0.0$ & 0.055 \\
\hline Days in hospital & $0.1 \pm 0.3$ & $0.0 \pm 0.0$ & NS* \\
\hline Oral steroid course & $0.8 \pm 1.1$ & $0.3 \pm 0.9$ & 0.036 \\
\hline Days housebound & $6.4 \pm 7.0$ & $0.5 \pm 1.3$ & 0.003 \\
\hline Asthma symptoms with exercise & $1.7 \pm 1.5$ & $0.6 \pm 1.1$ & 0.018 \\
\hline Health evaluation & $1.1 \pm 1.0$ & $0.5 \pm 0.7$ & 0.006 \\
\hline Energy evaluation & $0.9 \pm 1.1$ & $0.3 \pm 0.5$ & 0.02 \\
\hline $\begin{array}{l}\text { Mother's Depression Beck } \\
\text { Depression Inventory (BDI) }\end{array}$ & $16.2 \pm 10.6$ & $3.5 \pm 5.5$ & $<0.001$ \\
\hline
\end{tabular}

NS: Not significant 
Unscheduled doctor visits were reported by 10 of the 16 patients before the study, resulting in $1.8 \pm 1.8$ mean visits. Only one patient went to the doctor after bonding therapy $0.2 \pm 0.5(\mathrm{P}<0.001)$.

ER visits before treatment was reported by 4 out of 16 patients for an average of $0.4 \pm 1.0$ visits. No patient went to the ER post-treatment $0.0 \pm 0.0(\mathrm{P}=0.055)$.

Hospitalizations were reported by one child who spent 1 day in the hospital pre-treatment $0.1 \pm 0.3$. No patients were hospitalized after treatment. The sample size is insufficient to establish significance.

Oral Steroids were used by 7 patients before treatment, varying from 1 to 3 courses for an average of $0.8 \pm 1.1$ courses. Post-treatment, two patients required (a 2 and 3 course) oral steroid treatment $0.3 \pm 0.9$ $(\mathrm{P}=0.036)$.

Days Housebound were experienced by 8 patients pre-treatment $(4$ \pm 7.0 days $)$ and by 2 patients post-treatment $(0.5 \pm 1.3$ days, $\mathrm{P}=0.003)$.

Asthma symptoms from exercise were reduced from $(1.7 \pm 1.5$ to $0.6 \pm 1.1, \mathrm{P}=0.018)$.

Overall Health, as assessed by mothers improved after treatment $(1.1 \pm 1.0$ versus $0.5 \pm 0.7, \mathrm{P}=0.006)$.

Energy Level as assessed by mothers also improved after bonding therapy $(0.9 \pm 1.1$ versus $0.3 \pm 0.5, \mathrm{P}=0.020)$.

Medication: All 16 patients began the study on a variety of asthma maintenance medications, with some patients taking up to three different prescriptions. Post-treatment, 13 patients no longer required maintenance medication. Two of the three patients who remained on a medication were the older adolescents.

Beck Depression Inventory: In addition to the children's measurements, the mother's depression as scored on the Beck Depression Inventory (BDI) was collected before and after bonding therapy for 13 mothers. Prior to treatment the scores ranged from 3 to 37 (mean=16.2 \pm 10.6 ). Significant improvement was observed posttreatment with a range of 0 to $16($ mean $=3.5 \pm 5.5, \mathrm{P}<0.001)($ Table 2$)$.

All of the mothers DBI-II scores improved following treatment, even those of the mothers of the two older children, whose asthma showed no improvement. Two mothers jumped from being severely depressed to either mildly or minimally so. Six mothers changed from being mildly or moderately to minimally depressed. Four mothers

Table 2. Beck Depression Inventory_-II. Scores before and after treatment

\begin{tabular}{|c|c|c|c|c|c|}
\hline Pt ID & Age of child & Pre & Severity & Post & Severity \\
\hline 301 & 10 & 14 & Mild & 2 & Minimal \\
\hline 302 & 4.5 & 16 & Mild & 2 & Minimal \\
\hline 303 & 0.75 & 37 & Severe & 7 & Minimal \\
\hline 304 & 9 & 16 & Mild & 0 & Minimal \\
\hline 306 & 4.5 & 8 & Minimal & 0 & Minimal \\
\hline 307 & 11 & 16 & Mild & 2 & Minimal \\
\hline 309 & 6.5 & & & & \\
\hline 313 & 19 & 3 & Minimal & 0 & Minimal \\
\hline 314 & 15 & 3 & Minimal & 0 & Minimal \\
\hline 315 & 7 & 16 & Mild & 0 & Minimal \\
\hline 321 & 9 & & & & \\
\hline 323 & 2.5 & & & & \\
\hline 327 & 9 & 18 & Mild & 14 & Mild \\
\hline 328 & 6.5 & 35 & Severe & 15 & Mild \\
\hline 329 & 3 & 6 & Minimal & 1 & Minimal \\
\hline 331 & 11 & 23 & Moderate & 1 & Minimal \\
\hline
\end{tabular}

remained minimally depressed, although their scores were lower after treatment. Three mothers did not take the BDI-II.

\section{Discussion and conclusion}

This study, using more stringent measures of asthma symptoms, strengthens our previous findings that bonding a mother to her child seems to improve the child's asthma symptoms. With the exception of the two oldest adolescents, every child in the study showed improvement in all five categories of the Asthma Monitor: getting work done; shortness of breath; awakening at night; use of rescue inhaler; asthma out of control.

Every child in the study (except the two older adolescents) improved in the STEP measure of asthma severity, with the average moving down from "Moderate Persistent or Severe Persistent" to "No Asthma or Mild Intermittent." This improvement occurred without working directly with any of the children themselves but only with the mother.

There were fewer unscheduled doctor visits, fewer trips to the emergency room, and every child had fewer housebound days.

As we had discovered in our previous studies, this treatment does not seem to work for older adolescents. There has been speculation that this may be that bonding improvement has little effect on adolescents who are developmentally in the process of trying to separate from their parents.

We were surprised to find that bonding therapy improved the emotional state of every mother in the study, even mothers of the two older adolescents who did not improve. Some mothers, who were greatly depressed, improved considerably, with no other treatment than repairing the bond. None of the mothers received psychotherapy, but they responded as if they had. It appears that disrupted bonding may be responsible for some amount of depression in mothers, and that this is alleviated when the bond is repaired.

Because of concerns that asthma is often over-diagnosed, it would be useful in future studies to examine the incidence of bonding disruptions as well as the effectiveness of this treatment in all the different presentations of breathing problems in children.

The development of asthma has been linked to several types of maternal distress: psychological stress, health complications, mental health issues, care-giving problems, cesarean section deliveries, and pregnancy complications, among others. When examined through the lens of a maternal-infant bonding, they all fit the paradigm of "non-bonding events" [21]. Each of these can create in the mother an emotional state that is incompatible with bonding and can reduce the probability that bonding will occur [18]. It is our hypothesis that the mediating variable between these maternal distresses and asthma is the disruption in bonding between the mother and child. This hypothesis appears to be confirmed when we notice that the breathing problems improve once bonding is repaired.

How do disruptions in bonding lead to the subsequent expression of asthma and related breathing problems? Pennington [21] addressed this question when he reviewed the relationship between asthma and maternal issues. Summing up the literature in this area, he offers a hypothesis that a child's lack of connection with his mother "generates fear and its related behaviors, including endocrine and autonomic responses which lead to release of mediators responsible for bronchoconstriction". 
Mead [32] in reviewing separation upon the immune system and organ development writes:

Separation in early life is associated with changes in hypothalamicpituitary-adrenal (HPA) responses to stress, transient and long-term changes in immune competence in non-human primates and reduced maternal-infant attunement. The impact of maternal-infant separation during the sensitive period may permanently alter affectional ties, and may consequently influence developing organ systems, including the nervous system.

Klinnert, et al. [7] state that immune functioning in adults is known to be affected by stress and that this may alter the course of immune-based diseases such as allergies and asthma. So, too, parenting difficulties (i.e., resulting from bonding problems, in our way of thinking) may affect infants the way that stress affects adults and lead to a change in certain aspects of infant's immune system. Klinnert hypothesizes that the quality of care-giving affects the emotional and physiologic regulation of the infant and "could alter the hypothalamicpituitary-adrenal system or immune functioning in the direction of increased allergic response" [12].

Milam, et al. [33] write that the link between maternal stress and childhood stress is well known, and it seems apparent that childhood stress has a bearing on the endocrine system, the autonomic control of airways, and the immune system. Stress leads to the release of hormones that influence the immune system. Stress is linked to the secretion of adrenocorticotrophic hormone and cortisol, which increases the production of inflammatory cytokines. Therefore, it is possible that stress in a child can alter the functioning of the immune system which then causes inflammatory responses to triggers in the environment.

What greater stress could there be for a baby than to be chronically disconnected from its mother? We think that this stress in the unbonded child can lead, in some children, to inflammatory airway responses; and the present study seems to confirm this link.

\section{Acknowledgement}

The authors wish to thank the Association of Pre and Perinatal Psychology and Health, The Mental Insight Foundation, and the Huizenga Family for their generous contributions to this study.

\section{References}

1. Lara M, Rosenbaum S, Rachelefsky G, Nicholas W, Morton SC, et al. (2002) Improving childhood asthma outcomes in the United States: A blueprint for policy action. Pediatrics 109: 919-930.

2. Akinbami LJ, Moorman JE, Garbe PL, Sondik EJ (2009) Status of childhood asthma in the United States, 1980-2007. Pediatrics 123: 131-145. [Crossref]

3. DeFrances CJ, Cullen KA, Kozak LJ (2007) National hospital discharge survey: 2005 annual summary with detailed diagnosis and procedure data. Vital Health Stat 13: 1-209. [Crossref]

4. http://www.cdc.gov/VitalSigns/pdf/2011-05-vitalsigns.pdf

5. Martin M (2004) Asthma epidemic impacts Sonoma County. Sonoma Medicine 55.

6. Mrazek DA, Klinnert MD, Mrazek P, Macey T (1991). Early asthma onset: Consideration of parenting issues. J Am Acad Child Adolesc Psychiatry 30: 277-282. [Crossref]

7. Klinnert MD, Nelson HS, Price MR, Adinoff AD, Leung DY, et al. (2001) Onset and persistence of childhood asthma: Predictors from infancy. Pediatrics 108: E69. [Crossref]

8. Kero J, Gissler M, Grönlund MM, Kero P, Koskinen P, et al. (2002) Mode of delivery and asthma--Is there a connection? Pediatr Res 52: 6-11. [Crossref]

9. Roduit C, Scholtens S, de Jongste JC, Wijga AH, Gerritsen J, et al. (2009). Asthma at 8 years of age in children born by caesarean section. Thorax 64: 107-113. [Crossref]
10. Annesi-Maesano I, Moreau D, Strachan D (2001) In utero and perinatal complications preceding asthma. Allergy 56: 491-497. [Crossref]

11. Nafstad P, Samuelsen SO, Irgens LM, Bjerkedal T (2003) Pregnancy complications and the risk of asthma among Norwegians born between 1967 and 1993. Eur J Epidemiol 18: 755-761. [Crossref]

12. Kozyrskyj AL, Mai XM, McGrath P, Hayglass KT, Becker AB, et al. (2008) Continued exposure to maternal distress in early life increases the risk of childhood asthma. $\mathrm{Am} \mathrm{J}$ Respir Crit Care Med 177: 142-147. [Crossref]

13. Lange NE, Bunyavanich S, Silberg JL, Canino G, Rosner BA, et al. (2011) Parental psychosocial stress and asthma morbidity in Puerto Rican twins. J Allergy Clin Immunol 127: 734-740. [Crossref]

14. Mäntymaa M, Puura K, Luoma I, Salmelin R, Davis H, et al. (2003). Infant-mother interaction as a predictor of child's chronic health problems. Child Care Health Dev 29: 181-191. [Crossref]

15. Wright RJ, Cohen S, Carey V, Weiss SC, Gold DR (2002) Parental stress as a predictor of wheezing in infancy: A prospective birth-cohort study. Am J Respir Crit Care Med 165: 358-365. [Crossref]

16. Wright RJ, Visness CM, Calatroni A, Grayson MH, Gold DR, et al. (2010) Prenatal maternal stress and cord blood innate and adaptive cytokine responses in an inner-city cohort. Am J Respir Crit Care Med 182: 25-33. [Crossref]

17. Cassibba R, van IJzendoorn MH, Bruno S, Coppola G (2004) Attachment of mothers and children with recurrent asthmatic bronchitis. J Asthma 41: 419-431. [Crossref]

18. Klaus MH, Kennell JH (1976) Maternal-infant bonding. St. Louis, MO: C. V. Mosby.

19. Feinberg S (1988) Degree of maternal infant bonding and its relationship to pediatric asthma and family environments (Unpublished doctoral dissertation). The Professional School of Psychology, San Francisco, CA.

20. Schwartz M (1988) Incidence of events associated with maternal-infant bonding disturbances in a pediatric asthma population (Unpublished doctoral dissertation), Rosebridge Graduate School, Walnut Creek, CA.

21. Pennington D (1991) Events associated with maternal-infant bonding deficits and severity of pediatric asthma (Unpublished doctoral dissertation). The Professional School of Psychology, San Francisco, CA.

22. Madrid A, Ames R, Skolek S, Brown G (2000) Does maternal-infant bonding therapy improve breathing in asthmatic children? J Prenat Perinat Psychol Health 15: 90-112.

23. Madrid A, Ames R, Horner D, Brown G, Navarrette L (2004) Improving asthma symptoms in children by repairing the maternal-infant bond. J Prenat Perinat Psychol Health 18: 221-231.

24. American Psychiatric Association (2000) Diagnostic and statistical manual of mental disorders 4th ed., Text Revision Washington DC.

25. Martinez F (2002) Development of wheezing disorders and asthma in preschool children. Pediatrics 109: 362-367. [Crossref]

26. Kozyrskyj AL, Mustard CA, Becker AB (2003) Childhood wheezing syndromes and healthcare data. Pediatric Pulmonology 36: 131-136. [Crossref]

27. Brown G, Pennington D, Madrid A (1999) Maternal-infant bonding survey. Unpublished survey.

28. http://msdh.ms.gov/msdhsite/_static/resources/2100.pdf

29. Beck AT, Ward CH, Mendelson M, Mock J, Erbaugh J (1961). An inventory for measuring depression. Arch Gen Psychiatry 4: 561-571. [Crossref]

30. Madrid A (2005/2006) Helping children with asthma by repairing maternal-infant bonding problems. Am J Clin Hypn 48: 199-211. [Crossref]

31. Madrid A, Skolek S, Shapiro F (2006) Repairing failures in bonding through EMDR. Clinical Case Series 5: 271-286.

32. Mead V (2004) A new model for understanding the role of environmental factors in the origins of chronic illness. Med Hypotheses 63: 1035-1046. [Crossref]

33. Milam J, McConnell R, Yao L, Berhane K, Jerrett M, et al. (2008) Parental stress and childhood wheeze in a prospective cohort study. J Asthma 45: 319-323. [Crossref]

Copyright: (C2021 Madrid A. This is an open-access article distributed under the terms of the Creative Commons Attribution License, which permits unrestricted use, distribution, and reproduction in any medium, provided the original author and source are credited. 